\title{
Endoscopic versus conventional method for partial inferior turbinectomy in chronic hypertrophic rhinitis
}

\author{
Rai $S,{ }^{1^{*}}$ Sharma , $^{1}$ Koirala $K,{ }^{1}$ Sharma $A C^{1}$ \\ ${ }^{1}$ Department of ENT, Manipal Teaching Hospital, Pokhara, Nepal
}

*Corresponding Author:

Dr. Shiwani Rai, MS (ENT)

Lecturer

Department of ENT-HNS

Manipal Teaching Hospital, Pokhara, Nepal

E-mail: raishiwani@hotmail.com

\section{Citation}

Rai S, Sharma V, Koirala K, Sharma AC. Endoscopic versus conventional method for partial inferior turbinectomy in chronic hypertrophic rhinitis. Nepal Journal of Medical Sciences 2013;2(2):102-7.

\begin{abstract}
Background: The main goal of inferior turbinate surgery is to relieve obstruction while preserving the function of the turbinates as much as possible. Partial inferior turbinectomy (PIT) is an accepted and effective treatment in relieving the nasal obstruction while preserving the turbinate function. The use of endoscope provides complete visualization of the operating field and thus decreases the risk of excessive or inadequate resection.
\end{abstract}

Methods: A prospective single blinded randomized controlled trial was carried out in Department of ENT, Manipal Teaching Hospital, Pokhara, Nepal from September 2008 to March 2010 with an objective to compare between the conventional and endoscopic method of PIT in terms of outcome and post-operative morbidity in chronic hypertrophic rhinitis. Hundred cases were taken and assigned randomly into two groups; i.e. endoscopic PIT and conventional PIT. The post-operative findings were compared between the two groups using Fischer Exact test for proportion and $t$ test for mean.

Results: There was 94\% improvement in nasal obstruction in conventional group and $100 \%$ in endoscopic group at 1 month whereas improvement was $100 \%$ in both the groups at 3 months. Though no significant difference was seen statistically yet improvement was more in endoscopic group. There was significant difference in the SNOT 22 change score between endoscopic and conventional method at 1 month and 3 months. Significantly higher rates of post- operative morbidities were seen in case of conventional PIT at 1 month.

Conclusion: Endoscopic PIT is better than conventional in terms of improvement in nasal obstruction and decreased postoperative morbidity.

Keywords: Endoscopic; partial inferior turbinectomy; rhinitis; SNOT-22

\section{Background:}

Nasal obstruction, although not life threatening, poses a major problem for the patient and the operating surgeon. It interferes with the quality of life and is quite a challenging problem to deal with in terms of management. There is no single standardized mode of treatment that has evolved till now. ${ }^{1-3}$ The common cause of nasal obstruction in some patients may be hypertrophy of the inferior turbinate which may act independently or in conjunction with deviation of the septum and other rhinologic conditions. ${ }^{4}$ Chronic rhinitis is the common cause for inferior turbinate hypertrophy leading 
to nasal obstruction. The general term "chronic hypertrophic rhinitis" is used to describe the chronic rhinitis accompanied by turbinate enlargement refractory to nasal decongestants and characterized histologically by morphological changes of conchal mucosa, including squamous metaplasia with loss of cilia, fibrosis and goblet cell hyperplasia. ${ }^{5}$

Surgery for inferior turbinate hypertrophy was pioneered by Jones \& Holmes, ${ }^{6,7}$ since then a number of procedures has been described e.g. Complete or partial resection of the turbinates, sub mucous resection, radiofrequency ablation, laser turbinoplasty, cryosurgery, cauterization etc. Whatever the surgical technique may be; none of it is perfect and each of it is associated with long term or short term complications. The common complications encountered are epistaxis, atrophic rhinitis, crusting, synechiae formation and nasal discharge. $^{4}$

The main goal of inferior turbinate surgery in order to minimise the complications should be to relieve obstruction while preserving the function of the turbinates as much as possible. ${ }^{8}$ Partial turbinectomy is an accepted and effective treatment in relieving the nasal obstruction while preserving the turbinate function. The use of endoscope provides complete visualization of the operating field and thus decreases the risk of excessive or inadequate resection. In conventional partial turbinectomy the surgical exposure is limited, and visualization of the hypertrophied posteroinferior turbinate tip is difficult, especially in patients with small nostrils. Inadequate visualization of the surgical field can lead to incomplete resection of the postero-inferior turbinate and subsequently to persistent nasal obstruction. ${ }^{9,10}$

This study aims to compare the conventional surgical management i.e., partial inferior turbinectomy (PIT) without endoscopic guidance for chronic hypertrophic rhinitis with the newer endoscopic partial inferior turbinectomy in terms of improvement of nasal symptoms postoperatively and decrease in rate of various complications seen in conventional procedures.

\section{Methods:}

This is a prospective single blinded randomized controlled trial carried out in Department of Otorhinolaryngology, Manipal Teaching Hospital, Pokhara, Nepal from September 2008 to March 2010 with an objective to compare between the conventional and endoscopic methods of partial inferior turbinectomy in terms of outcome and post-operative morbidity for cases with chronic hypertrophic rhinitis. The total number of turbinate surgery done in this hospital in last 18 months before commencing this study was 120 .
Taking 95\% confidence level and 5\% confidence interval, the sample size was calculated to be 92 using a sample size calculator from www.surveysystem.com. So we took 100 cases for the study and divided it into two equal groups; i.e. 50 for endoscopic PIT and 50 for conventional PIT group. All patients included in the study were above 15 years and less than 45 years with bilateral hypertrophied inferior turbinates and features of nasal obstruction for more than 3 months which was refractory to nasal decongestants. Presence of any active infection, bleeding disorders and pre-existing nasal dryness or crust formation were also ruled out before enrolment of the cases for the study. The selected patients were requested to fill up the SNOT-22 (Sino nasal outcome test-22) questionnaires which was read out and translated to them. All cases meeting the inclusion criteria were included in the study and were randomly assigned to Conventional method and Endoscopic method. Informed consent was taken from all the patients before enrolling them for the study and those who denied giving the consent or opted for treatment other than that was designated to them according to the randomisation were also excluded from the study.

Endoscopic PIT was conducted under general anaesthesia using a 30 degree rigid nasal endoscope. Nasal cavities was packed (with ribbon gauze soaked in $4 \%$ Lignocaine mixed with 1 in 1 lakh adrenaline) for 10 minutes before surgery. Nasal packs were removed and the inferior turbinates infiltrated with $2 \%$ Lignocaine mixed with 1 in 2 lakh adrenaline. Inferior turbinate was then in-fractured medially with Freer's elevator. The turbinate was clamped for 1 minute with straight artery forceps placed along its length near its insertion into the lateral nasal wall. Inferior turbinate was then trimmed along the crushed line with Blakesley's nasal scissors. This being smaller than the conventional turbinectomy scissors is easy to manipulate beneath the turbinate. The turbinate was usually removed as a single piece. The cut edges of turbinate remnant were cauterized meticulously using the bipolar cautery under endoscopic guidance. The cauterization was done in anterior to posterior direction. This allows existing bleeding to flow posteriorly and the source of bleeding is visualized easily. Deviated nasal septum if presented was corrected with septoplasty done by Cottle's technique. After securing haemostasis, both nasal cavities were packed with $1 / 2$ inch gauze piece soaked in paraffin + antibiotic cream.

Conventional PIT was also carried out similarly under general anaesthesia using headlight but without the endoscope. Nasal packs were removed gently after 48 hours and checked for bleeding. The patients were discharged the 
next day and asked to follow up after 1 and 3 months of surgery.

At follow up patients were questioned and examined regarding the presence of nasal obstruction, excessive sneezing, nasal discharge, dryness of nasal cavity, nasal crusting, synechiae and foetor (foul smell) at 1 month and 3 months after the surgery. A through ENT examination as well as a Diagnostic Nasal Endoscopy (DNE) was done in each visits. They were also requested to fill up the SNOT- 22 questionnaires at each visit to assess improvement.

The collected data were statistically analysed using SPSS 11.5 software. The post-operative findings were compared between the two groups using Fischer Exact test for proportion and $\mathrm{t}$ test for mean.

\section{Results:}

The mean age of the conventional group was 24.76 years with a range from 17-45 years and S.D of 7.91. The mean age of endoscopic group was 27.46 years with a range from $17-45$ years and S.D of 9.14 . There were $72 \%$ male and $28 \%$ female $(\mathrm{M}: \mathrm{F}=2.57: 1)$ in conventional group and 70\% male and 30\% female $(\mathrm{M}: \mathrm{F}=2.33: 1)$ in endoscopic group.

Preoperatively all the patients had nasal obstruction in both the groups. Thirty six (76\%) and 32 (64\%) of the patients in conventional and endoscopic group had excessive sneezing. Forty four (88\%) and 46 (92\%) of the cases in conventional and endoscopic group respectively had deviated nasal septum. Both the groups were matched showing no significant statistical difference in terms of age, sex and preoperative findings (Table 1).

Table 1: Showing age, sex and preoperative findings in both the group

\begin{tabular}{lccc}
\hline \multicolumn{1}{c}{ Parameters } & $\begin{array}{c}\text { Conventional } \\
\text { group }\end{array}$ & $\begin{array}{c}\text { Endoscopic } \\
\text { Group }\end{array}$ & p value \\
\hline Mean Age & 24.76 & 27.46 & 0.118 \\
Mex $\quad$ Male & 36 & 35 & 1 \\
$\quad$ Female & 14 & 15 & 1 \\
Nasal obstruction & $50(100 \%)$ & $50(100 \%)$ & 1 \\
Sneezing & $38(76 \%)$ & $32(64 \%)$ & 0.28 \\
Nasal Discharge & $0(0 \%)$ & $0(0 \%)$ & 1 \\
DNS & $44(88 \%)$ & $46(92 \%)$ & 0.74 \\
\hline
\end{tabular}

After the removal of nasal pack at 48 hours, bleeding was seen in $10(20 \%)$ of cases in conventional group whereas there was no bleeding in cases of endoscopic group ( $p$ $<0.001)$.

After 1 month of surgery, there was 94\% improvement in nasal obstruction in case of conventional group whereas there was $100 \%$ improvement in case of endoscopic group $(\mathrm{p}=0.24)$. Excessive sneezing was still present in $54 \%$ of cases of conventional group whereas in endoscopic group it was present only in $8 \%$ of cases $(\mathrm{P}<0.001)$. There was no crusting and synechiae in case of endoscopic group whereas there was $60 \%$ crusting $(\mathrm{P}<0.001)$ and $12 \%$ synechiae $(p=0.03)$ seen in cases of conventional group. Nasal discharge and foetor were however absent in both the groups at 1 month (Table 2).

Table 2: Post- operative findings in conventional and endoscopic groups

\begin{tabular}{|c|c|c|c|c|c|c|}
\hline \multirow[b]{2}{*}{ Findings } & \multicolumn{3}{|c|}{1 Month Post-op } & \multicolumn{3}{|c|}{3 months Post-op } \\
\hline & $\begin{array}{c}\text { Conven- } \\
\text { tional (\%) }\end{array}$ & $\begin{array}{c}\text { Endo- } \\
\text { scopic (\%) }\end{array}$ & 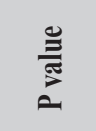 & $\begin{array}{c}\text { Conven- } \\
\text { tional } \\
(\%)\end{array}$ & $\begin{array}{c}\text { Endo- } \\
\text { scopic } \\
(\%)\end{array}$ & 总 \\
\hline $\begin{array}{l}\text { Nasal } \\
\text { obstruction }\end{array}$ & $3(6)$ & $0(0)$ & 0.24 & $0(0)$ & $0(0)$ & 1 \\
\hline Sneezing & $27(54)$ & $4(8)$ & $<0.001$ & $5(10)$ & $4(8)$ & 0.74 \\
\hline $\begin{array}{l}\text { Nasal } \\
\text { discharge }\end{array}$ & 0 & 0 & 1 & $0(0)$ & $0(0)$ & 1 \\
\hline Foetor & $0(0)$ & $0(0)$ & 1 & $0(0)$ & $0(0)$ & 1 \\
\hline Crusting & $30(60)$ & $0(0)$ & $<0.001$ & $2(4)$ & $0(0)$ & 0.49 \\
\hline Synechiae & $6(12)$ & $0(0)$ & 0.03 & $1(2)$ & $0(0)$ & 1 \\
\hline
\end{tabular}

At 3 months of surgery, there was $100 \%$ improvement in nasal obstruction in both the groups $(p=1)$. Excessive sneezing was still present in $10 \%$ of cases of conventional group whereas it was present only in $8 \%$ of cases in endoscopic group $(\mathrm{P}=0.74)$. Similarly there was no crusting and synechiae in case of endoscopic group whereas there was $4 \%$ crusting $(\mathrm{P}=0.49)$ and $2 \%$ synechiae $(\mathrm{p}=1)$ seen in cases of conventional group. Foetor and nasal discharge was absent in both the groups at 3 months as well (Table 2).

The mean SNOT 22 score at presentation in endoscopic group was 39.42 and it improved to 10.30 at 1 month and 5.14 at 3 months. In conventional group the mean SNOT 22 at presentation was 32.10 and it improved to 13.98 at 1 month and 9.04 at 3 months. There was significant difference 
between pre-operative and post-operative SNOT 22 score in both conventional and endoscopic group at 1 month and 3 months as well $(\mathrm{P}<0.001)$ (Table 3$)$.

Table 3: showing significant difference between pre-op and post op SNOT at 1 and 3 months using paired t test

\begin{tabular}{|c|c|c|c|c|c|c|c|}
\hline Pair & Mean & SD & SEM & 总 & df & 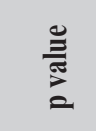 & $\begin{array}{c}95 \% \text { CI } \\
\text { of the } \\
\text { difference }\end{array}$ \\
\hline $\begin{array}{l}\text { E SNOT1- } \\
\text { E SNOT2 }\end{array}$ & 29.12 & 8.42 & 1.19 & 24.44 & 49 & $<0.001$ & $26.73-31.51$ \\
\hline $\begin{array}{l}\text { C SNOT1- } \\
\text { C SNOT2 }\end{array}$ & 18.12 & 7.18 & $1 . / 2$ & 17.86 & 49 & $<0.001$ & $16.08-20.16$ \\
\hline $\begin{array}{l}\text { E S N O T } 1 \text { - } \\
\text { ESNOT3 }\end{array}$ & 34.28 & 9.31 & 1.32 & 26.05 & 49 & $<0.001$ & $31.64-36.92$ \\
\hline $\begin{array}{l}\text { C SNOT1- } \\
\text { C SNOT3 }\end{array}$ & 23.06 & 6.74 & 0.95 & 26.05 & 49 & $<0.001$ & $31.64-36.92$ \\
\hline
\end{tabular}

The mean SNOT 22 change score at 1 month was 29.04 (SD 8.54) in endoscopic group and 18.32 (SD 7.08) in conventional group and the difference was statistically significant $(\mathrm{p}<0.001)$. Similarly at 3 months the mean SNOT 22 change score was 34.24 (SD 9.35) in endoscopic group and 23.46 (SD 6.67) in conventional group and the difference was statistically significant $(\mathrm{p}<0.001)$ (Table 4).

Table 4: showing statistically significant difference in SNOT 22 change score at 1 and 3 months between the two groups.

\begin{tabular}{|c|c|c|c|c|c|c|c|}
\hline Pair & Mean & SD & SEM & $\frac{\mathscr{E}}{\underline{\Xi}}$ & df & 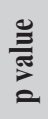 & $\begin{array}{c}95 \% \text { CI } \\
\text { of the } \\
\text { difference }\end{array}$ \\
\hline
\end{tabular}

E SNOT CH1-
C SNOT CH1

ESNOT CH3-

$\begin{array}{llllllll}\text { C SNOT CH3 } & 10.78 & 10.78 & 1.53 & 7.07 & 49 & <0.001 & 7.72-13.84\end{array}$

\section{Discussion:}

Partial inferior turbinectomy is an established and effective modality of treatment in refractory chronic hypertrophic rhinitis. However complications like bleeding ranging from minimal bleeding to life threatening bleeding requiring transfusions, crusting, dryness and synechiae has been reported. Endoscopic reduction of inferior turbinate using powered microdebrider was described by Bielamowicz et al in 1991. ${ }^{9}$ The use of endoscope further decreased the morbidity allowing better visualization of the operative field and allowing precise reduction of the inferior turbinate. ${ }^{9}$ The risk of incomplete resection of the posterior inferior turbinate is overcome by the use of endoscope. Complete operative visualisation enhances accurate resection of the inferior and lateral inferior turbinate bone and mucosa thereby minimizing any chances of bleeding.

We have performed the same old technique of partial inferior turbinate reduction using an endoscope but without any use of powered instruments like microdebrider to study and compare the postoperative morbidity along with bleeding and improvement of symptoms of obstruction compared to conventional partial inferior turbinectomy. We have also used SNOT-22, a validated tool for assessing the outcome in sinonasal disorders, to assess the subjective improvement in patients postoperatively.

Improvement in nasal obstruction was seen in $100 \%$ of cases both at 1 month and at 3 months in endoscopic group whereas in conventional PIT group, improvement was seen in $94 \%$ of cases at 1 month $(\mathrm{p}=0.24)$ and $100 \%$ at 3 months $(p=1)$. This shows that though not statistically significant endoscopic approach has more benefit in early improvement of nasal obstruction than conventional. Studies in the recent past have reported improvement in nasal obstruction 77$100 \% .^{3,9,11-14}$ A significant difference in pre-op and post-op SNOT-22 score at both 1 month and 3 months was seen in cases of endoscopic group and conventional group. The mean SNOT 22 score at presentation in endoscopic group was 39.42 and it improved to 10.30 at 1 month $(\mathrm{p}<0.001)$ and 5.14 at 3 months $(\mathrm{p}<0.001)$ showing statistically significant improvement. In conventional group the mean SNOT 22 at presentation was 32.10 and it improved to 13.98 at 1 month $(p<0.001)$ and 9.04 at 3 months $(p<0.001)$ showing significant improvement. This shows that both these procedures are well effective in treating hypertrophic rhinitis as there was significant change in pre-treatment and post-treatment SNOT values in both cases. The mean SNOT 22 change score at 1 month was 29.04 in endoscopic group and 18.32 in conventional group $(\mathrm{p}<0.001)$ whereas at 3 months the mean SNOT 22 change score was 34.24 in endoscopic group and 23.46 in conventional group $(\mathrm{p}<0.001)$ showing statistically significant difference. This shows that the improvement in nasal outcome, as suggested by SNOT 22 change score, was significantly high in endoscopic group than in conventional group. There are some studies in inferior turbinectomy which have used non validated questionnaires or other validated tools (VAS) ${ }^{15}$ to 
evaluate the outcome postoperatively but none of them have used SNOT tool as in our study.

Postoperative bleeding was assessed at 48 hours after pack removal. We found no bleeding in cases of endoscopic group whereas there was bleeding in $20 \%$ of cases $(p<0.001)$ in conventional group showing statistically significant difference. All of these cases were managed by anterior nasal packing for 48 hours and required no further interventions. Bleeding has been reported from 3 to $12 \%$ in cases of inferior turbinate surgery. ${ }^{3,11-14}$ In case of endoscopic partial inferior turbinectomy reported by Bielamowicz et $\mathrm{al}^{9}$ there was $95 \%$ bleeding during pack removal for less than 5 minutes, $5 \%$ bleeding between 5-15 minutes and no bleeding for more than 15 minutes. None of these cases required packing or other interventions.

There was no crusting in case of endoscopic group whereas there was $60 \%$ crusting $(\mathrm{P}<0.001)$ seen in cases of conventional group at 1 month post op showing statistically significant difference in crusting between the two groups. Similarly there was no crusting in case of endoscopic group at 3 months whereas there was $4 \%$ crusting $(\mathrm{P}=0.49)$ in cases of conventional group showing no significant difference. Crusting was reduced with nasal douching. Most of the authors have reported crusting in inferior turbinate surgery from $0 \%$ to $13 \% .^{3,11-14}$ In endoscopic PIT, Bielamowicz et al ${ }^{9}$ have reported that $60 \%$ of cases had mild amount of crusting in immediate post op period which resolved by $3^{\text {rd }}$ week. At 6 months follow up there was no crusting seen in these cases.

There was no synechiae in case of endoscopic group whereas there was $12 \%$ synechiae $(p=0.03)$ seen in cases of conventional group at 1 month showing statistically significant difference in synechiae seen in these two procedures. Similarly there was no synechiae in case of endoscopic group whereas there was $2 \%$ synechiae $(p=1)$ seen in cases of conventional group at 3 months showing no significant difference. The synechiae was released in OPD and a wax plate was placed for 5 days to prevent further synechiae. 3-4.4\% synechiae has been reported in the literature by various authors. ${ }^{3,11-14}$ In case of endoscopic partial inferior turbinectomy reported by Bielamowicz et $\mathrm{al}^{9}$ found $10 \%$ mild asymptomatic synechiae which resolved without any complications. There was no synechiae seen in these cases at 3 months follow up.

Nasal discharge and foetor was absent in both the groups in our study. 5-39\% of nasal discharge has been observed in other series in the past literatures in inferior turbinate surgery. ${ }^{3,11-14}$ In case of endoscopic partial inferior turbinectomy reported by Bielamowicz et $\mathrm{al}^{9}$ has also reported no incidences of these complications.

\section{Conclusion:}

The SNOT-22 change score was significantly higher in endoscopic group than conventional group at both 1 month and 3 months after surgery. Improvement in nasal obstruction was seen more in endoscopic group than in conventional group for PIT. The incidence of immediate post-operative bleeding, presence of sneezing, crusting and synechiae were also seen significantly less in case of endoscopic PIT. This suggests that endoscopic PIT is better and safe procedure than conventional PIT in treating chronic hypertrophic rhinitis.

\section{References:}

1. Lippert BM, Werner JA. $\mathrm{CO}_{2}$ laser surgery of hypertrophied inferior turbinates. Rhinology 1997;35:33-6.

2. Goldsher M, Joachims HZ, Golz A. Nd:YAG laser turbinate surgery animal experimental study: preliminary report. Laryngoscope 1995;105:319-21.

3. Rakover Y, Rosen G. A comparison of partial turbinectomy and cryosurgery for hypertrophic inferior turbinates. J Laryngol Otol 1996;110:732-5.

4. Huang T, Cheng P. Changes in nasal resistance and quality of life after endoscopic microdebrider assisted inferior turbinoplasty in patients with perennial allergic rhinitis. Arch Otolaryngol Head Neck Surg 2006;132:990-3.

5. Berger G, Gass S, Ophir D .The histopathology of the hypertrophic inferior turbinate. Arch Otolaryngol Head Neck Surg 2006;132:588-94.

6. Friedman M, Tanyeri H, Lim J, et al. A safe, alternative technique for inferior turbinate reduction. Laryngoscope 1999;109:1834-7.

7. Bielamowicz S, Hawrych A, Gupta A. Endoscopic inferior turbinate reduction: a new technique. Laryngoscope 1999;109:1007-9.

8. Lee C, Chen T. Power Microdebrider-Assisted Modification of Endoscopic Inferior Turbinoplasty: A Preliminary Report. Chang Gung Med J 2004;27:359-64.

9. Kafle P, Maharjan M, Sherestha S, et al. Comparison of sub mucosal diathermy and partial resection of Inferior turbinate in the treatment of symptomatic nasal valve 
Original Article | Rai S, et al. Endoscopic partial inferior turbinectomy in chronic hypertrophic rhinitis

blockage. Kathmandu Univ Med J 2007;5:501-3.

10. Jones, TC. Turbinotomy. Lancet 1895;2:496.

11. Holmes CR. Hypertrophy of the turbinated bodies. N Y Med J 1900;72: 529.

12. Martinez SA, Nissen AJ, Stock CR, et al. Nasal turbinate resection for relief of nasal obstruction. Laryngoscope 1983;93:871-5.

13. Mucci S, Sismanis A. Inferior partial turbinectomy: an effective procedure for chronic rhinitis. ENT Journal 1994;73:405-7.
14. Mathai J: Inferior turbinectomy for nasal obstruction review of 75 cases. Indian J otolaryngol Head Neck Surg 2004;56:23-6.

15. Manzoor T, Asbhar A, Aslam S, et al. Partial inferior turbinectomy; a better management option for hypertrophied inferior turbinates. Professional Med J 2008;15:512-7. 